\title{
Scale-dependent colocalization in a population of gyrotactic swimmers
}

\author{
M. Borgnino, F. De Lillo, and G. Boffetta \\ Department of Physics and INFN, Università di Torino, via P. Giuria 1, I-10125 Torino, Italy
}

(Received 7 December 2016; published 17 February 2017)

\begin{abstract}
We study the small scale clustering of gyrotactic swimmers transported by a turbulent flow, when the intrinsic variability of the swimming parameters within the population is considered. By means of extensive numerical simulations, we find that the variety of the population introduces a characteristic scale $R^{*}$ in its spatial distribution. At scales smaller than $R^{*}$ the swimmers are homogeneously distributed, while at larger scales an inhomogeneous distribution is observed with a fractal dimension close to what observed for a monodisperse population characterized by mean parameters. The scale $R^{*}$ depends on the dispersion of the population and it is found to scale linearly with the standard deviation both for a bimodal and for a Gaussian distribution. Our numerical results, which extend recent findings for a monodisperse population, indicate that in principle it is possible to observe small scale, fractal clustering in a laboratory experiment with gyrotactic cells.
\end{abstract}

DOI: 10.1103/PhysRevE.95.023108

\section{INTRODUCTION}

The microscopic distribution of aquatic microorganisms has profound effects on the ecology of the oceans [1,2]. One example is the observed patchiness of phytoplankton at the submeter scale which has a fundamental impact on the rate at which cells encounter each other and their predators $[3,4]$. Patchiness of phytoplankton at different scales has different origins. While at large scales it is driven by reproduction and/or nutrients $[5,6]$, at scales smaller than one kilometer patchiness is expected to be produced by physical mechanisms, including plankton motility and the interaction with the flow. Indeed, field observations have revealed that motile phytoplankton are considerably more patchy at small scales than nonmotile species $[7,8]$.

Several species of motile phytoplankton are able to swim in the vertical direction guided by a stabilizing torque arising from an unbalance distribution of the mass in the cell $[9,10]$. The resulting swimming direction of these gyrotactic cells stems from the competition between the stabilizing torque and the shear-induced viscous torque [11-14]. Numerical and experimental works have revealed how gyrotactic motility, combined with the presence of a flow, generates strongly inhomogeneous distributions. In the case of laminar flow, gyrotaxis produces a beamlike accumulation in downwelling pipe flows [11], while in horizontal shear flow it generates accumulation in thin layers $[10,15,16]$. Recent works have shown that gyrotaxis also produces clustering at very small scales (comparable with the Kolmogorov scale) in nonstationary turbulent flows [17-20]. In this case cells are found to accumulate on fractal dynamical clusters characterized by a fractal dimension which depends on the cell and flow parameters [17,18,21].

In this work we consider the dynamics of an inhomogeneous population of gyrotactic cells, characterized by a distribution of cells' parameters, transported by a turbulent flow in the limit of dilute concentration, i.e., neglecting the interaction among cells and possible feedback on the flow. The motivation of our study is to determine the robustness of fractal clustering induced by turbulence on a distribution of cells with slightly different biological parameters, typical of a natural population. The main result, obtained by means of extensive numerical simulations, is that fractal clustering is observable, at large enough scales, also in populations with significant variability (up to $20 \%$ of relative variation in gyrotactic parameters). Moreover, by considering a simplified bimodal population, we introduce a crossover scale (above which fractal clustering is observable) and we predict how this scale depends on the population variability.

The remaining part of this paper is organized as follows. In Sec. II we introduce the mathematical model for gyrotactic swimmers and we discuss, on the basis of simple arguments, how clustering depends on the population distribution. Section III is devoted to numerical results for two particular distributions, while Sec. IV summarizes our results.

\section{MATHEMATICAL MODEL}

We consider the classical model of gyrotactic swimmers which describes the motion of a bottom-heavy spherical cell [11,22] at position $\boldsymbol{X}$ swimming in the direction $\boldsymbol{p}$ (with $|\boldsymbol{p}|=1)$,

$$
\begin{gathered}
\frac{d \boldsymbol{X}}{d t}=\boldsymbol{u}(\boldsymbol{X}, t)+V \boldsymbol{p}, \\
\frac{d \boldsymbol{p}}{d t}=\frac{1}{2 B}[\boldsymbol{k}-(\boldsymbol{k} \cdot \boldsymbol{p}) \boldsymbol{p}]+\frac{1}{2} \omega(\boldsymbol{X}, t) \times \boldsymbol{p},
\end{gathered}
$$

where $\boldsymbol{u}(\boldsymbol{x}, t)$ is the velocity field, $\boldsymbol{\omega}=\boldsymbol{\nabla} \times \boldsymbol{u}$ is the vorticity, $\boldsymbol{k}=(0,0,1)$ is the vertical unit vector. The first term on the right-hand side of (2) represents the effect of the gravitational torque which orients the swimming direction towards the vertical, while the last term is viscous torque which rotates the cells with the local vorticity. $V$ is the swimming velocity, assumed constant, while $B=3 \mathrm{v} /(\mathrm{gh})$ is the gyrotactic reorientation time where $v$ is the kinematic viscosity of the fluid, $g$ the acceleration of gravity, and $h$ measures the displacement of the center of mass from the geometrical center of the cell.

The gyrotactic swimmers are transported by a turbulent velocity field $\boldsymbol{u}(\boldsymbol{x}, t)$ obtained by direct numerical simulations (DNS) of the incompressible Navier-Stokes equations,

$$
\partial_{t} \boldsymbol{u}+\boldsymbol{u} \cdot \nabla \boldsymbol{u}=-\nabla p+v \nabla^{2} \boldsymbol{u}+\boldsymbol{f},
$$


where $\boldsymbol{f}$ represents a zero-mean, temporally uncorrelated Gaussian forcing which injects energy at large scales at a rate $\varepsilon$. Together with the viscosity, the energy injection rate defines the Kolmogorov length scale $\eta_{K}=\left(v^{3} / \varepsilon\right)^{1 / 4}$, the Kolmogorov time scale $\tau_{K}=(v / \varepsilon)^{1 / 2}$, and the Kolmogorov velocity $v_{K}=$ $\eta_{K} / \tau_{K}=(\nu \varepsilon)^{1 / 4}$ [23]. These characteristic scales are used to make the parameters in the gyrotactic model dimensionless. The ratio of the two terms on the right-hand side of (1) defines the swimming number $\Phi \equiv V / v_{K}$, while the ratio of the two terms in Eq. (2) gives the stability number $\Psi \equiv B / \tau_{K}$.

Formally, Eqs. (1) and (2) define a dissipative dynamical system in the $(\boldsymbol{X}, \boldsymbol{p})$ phase space of dimension $2 d-1(d=3)$ with an expansion rate in the phase space given by

$$
\sum_{i=1}^{d}\left(\frac{\partial \dot{X}_{i}}{\partial X_{i}}+\frac{\partial \dot{p}_{i}}{\partial p_{i}}\right)=-\frac{d-1}{2 B} p_{3} .
$$

As the swimming direction orients towards the vertical direction $\left(p_{3}>0\right)$ the expansion rate becomes negative and the trajectories collapse on a fractal attractor in the phase space. When the attractor has dimension less than $d$ the swimmers concentrate (in physical space) on clusters with the same fractal dimension [24].

When the swimming number vanishes (i.e., $V=0$ ) the cells in Eq. (1) are simply transported by an incompressible velocity field and therefore they cannot accumulate [as (1) decouples from (2)]. Moreover, when $B$ is smaller than the Kolmogorov time, i.e., $\Psi \ll 1$, we can expand (2) at the first order in $B / \tau_{K}$ to obtain, in stationary conditions [17],

$$
\boldsymbol{p} \approx\left(B \omega_{y},-B \omega_{x}, 1\right),
$$

which shows that when $B=0, \boldsymbol{p}$ is aligned towards the vertical direction and the motion of the swimmers is given by the superposition of an incompressible velocity and a uniform vertical migration which, again, cannot produce clustering. Similarly, for $B \rightarrow \infty$ the expansion rate (4) vanishes and also in this case swimmers are not expected to cluster. Previous numerical simulations have shown that indeed gyrotactic swimmers produce clusters for intermediate values of $\Psi$ (and $\Phi>0$ ) with maximum clustering for $\Psi \simeq 1$ [17].

To quantify the degree of clustering we use the correlation dimension $D$, defined as the scaling exponent of the probability to find two particles at a distance less than $r: P\left(\left|X_{1}-X_{2}\right|<\right.$ $r) \propto r^{D}$ as $r \rightarrow 0$ [24]. For homogeneous distribution in space one has $D=d$, while $D<d$ indicates fractal clustering.

When considering a population of swimmers with different parameters $V$ and $B$ we can extend the above definition to measure the cross-correlation dimension $D_{12}(r)$ defined in terms of the probability of finding two swimmers characterized by two sets of parameters $\left(V_{1}, B_{1}\right)$ and $\left(V_{2}, B_{2}\right)$ at a distance smaller than $r: P_{12}(r) \propto r^{D_{12}}$ [25]. In principle, we cannot expect a power-law scaling for $P_{12}(r)$ for a generic couple of swimmer parameters and therefore $D_{12}$ is a function of $r$ and not simply a scaling exponent. Of course, for a monodisperse population, with $V_{2}=V_{1}$ and $B_{2}=B_{1}$ the cross-correlation dimension recovers the correlation dimension of the population, $D_{12}(r)=D$.

Consider now a couple of swimmers at positions $\boldsymbol{X}_{1}$ and $\boldsymbol{X}_{2}=\boldsymbol{X}_{1}+\boldsymbol{R}$ with slightly different parameters, e.g., with the same swimming velocity $V_{2}=V_{1}$ and with different reorientation time $B_{2}=B_{1}+\Delta B$. We assume that $\Delta B$ is a small parameter such that the separation between the two trajectories $\boldsymbol{R}$ is smaller than the Kolmogorov scale. According to (1) this separation evolves according to

$$
\frac{d \boldsymbol{R}}{d t}=\Delta \boldsymbol{u}(\boldsymbol{R})+V \Delta \boldsymbol{p},
$$

where $\Delta \boldsymbol{u}(\boldsymbol{R})=\boldsymbol{u}\left(\boldsymbol{X}_{2}\right)-\boldsymbol{u}\left(\boldsymbol{X}_{1}\right)$ and $\Delta \boldsymbol{p}=\boldsymbol{p}_{2}-\boldsymbol{p}_{1}$.

The first term on the right-hand side of (6) is proportional to $v_{K}\left(R / \eta_{K}\right)$, while the second term, in the limit of small stability numbers, contains the difference $\omega \Delta B$. The ratio of these two terms defines a characteristic scale $R^{*} \simeq \eta_{K} \Phi \Delta \Psi$. When $R<R^{*}$ the swimmer velocity difference is dominated by the second term in Eq. (6): The two trajectories are uncorrelated and one swimmer sees the other population as uniformly distributed. On the contrary, when $R>R^{*}$, the first term in Eq. (6) dominates and correlations between the two populations appear, induced by the common velocity field [26]. Therefore, for a bimodal distribution, we expect two different behaviors for $D_{12}(r): D_{12}(r)=3$ for $R<R^{*}$ and $D_{12}(r) \simeq$ $D_{11}$ for $R>R^{*}\left(D_{11} \simeq D_{22}\right.$ are the correlation dimensions of the two populations, which are close by hypothesis).

In the case of two swimmers with the same reorientation time $B$ and different swimming velocity $V_{1}$ and $V_{2}=V_{1}+$ $\Delta V$, a similar argument, in the limit of small parameter difference, leads again to a characteristic scale $R^{*} \simeq \eta_{K} \Psi \Delta \Phi$ which separates scales with homogeneous and fractal relative distribution.

The general case of a polydisperse population, characterized by a probability density function of parameters $f(V, B)$ is the most interesting for applications to experimental data where one cannot avoid the natural intrinsic variability of the population. Also in this case we will consider the cumulative probability of having two swimmers at a distance lower than $r$, integrated over the distribution $f(V, B)$. Again, for very small $r$ we expect this probability to decrease proportional to $r^{3}$ as different cells in the population are spatially decorrelated. The interesting question is whether also for a continuous distribution of cell parameters with finite support there exists a characteristic scale $R^{*}$ above which a fractal dimension can be observed which can be interpreted as that of an "average" monodisperse population. To address this point we will consider a population characterized by a Gaussian parameter distribution $f(B)$ with mean value $\bar{B}$ and variance $\sigma_{B}$.

\section{NUMERICAL RESULTS}

We have performed a numerical investigation of the spatial distribution of several populations of swimmers, characterized by different distributions $f(V, B)$ of swimming parameters. The velocity (and vorticity) field in (1) and (2) are obtained by a direct numerical simulation of the NS equations (3) by using a fully dealiased pseudospectral code at different resolutions. After the flow has reached a statistical steady state, a population of $N_{s}$ cells is initialized with uniform random positions $\boldsymbol{X}$ in the domain and orientation $\boldsymbol{p}$ on the unit sphere. The motion of the swimmers is obtained by the simultaneous integration of (3) and (1) and (2) in which fluid velocity and vorticity at the cell positions are obtained by trilinear 


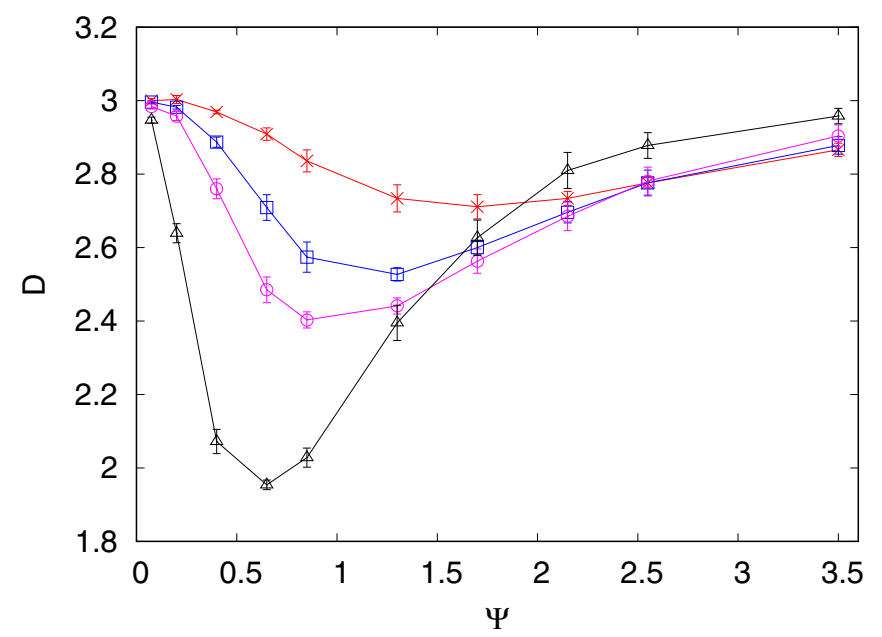

FIG. 1. Correlation dimension $D$ for a homogeneous population of gyrotactic swimmers as a function of the stability number $\Psi$. Different lines correspond to different swimming numbers: $\Phi=0.33$ (red crosses), $\Phi=0.66$ (blue squares), $\Phi=1.0$ (purple circles), and $\Phi=3.0$ (black triangles). The error bars are estimated on the fluctuations of the dimension with the statistics.

interpolation [27]. After the swimmer distribution has reached a statistical steady state, we collect data for several large-scale eddy turnover times to ensure statistical convergence.

In Fig. 1 we plot the correlation dimension for a monodisperse population as a function of the swimming number $\Phi$ and stability number $\Psi$. As already reported, clustering is maximum (i.e., $D$ is minimum) for $\Psi \simeq 1$ and large $\Phi$ $[17,20]$ while $D \simeq 3$ for both large and small values of $\Psi$, as discussed in Sec. II. The position of the minimum $D$ (maximum clustering) depends on the swimming velocity as, for small $\Psi$, one has $3-D \propto(\Phi \Psi)^{2}[17]$.

\section{A. Bimodal distribution}

We first consider a bimodal population composed by two species with the same swimming number $\Phi$ and different stability numbers $\Psi_{1}$ and $\Psi_{2}=\Psi_{1}+\Delta \Psi$ with the same number of cells in each species, i.e., with marginal distribution $f(B)=\frac{1}{2} \delta\left(B-B_{1}\right)+\frac{1}{2} \delta\left(B-B_{2}\right)$. The difference $\Delta \Psi$ defines the standard deviation of the distribution $\sigma_{B}=\Delta \Psi / 2$.

Figure 2 shows a two-dimensional (2D) section of the three-dimensional (3D) distribution of a bimodal population with dimensionless parameters $\Psi_{1}=0.5, \Psi_{2}=0.667$, and $\Phi_{1}=\Phi_{2}=3.0$ with the two species plotted with different color. Both species are expected to cluster according to the results shown in Fig. 1 with correlation dimension $D \simeq 2.0$. It is evident that the two populations display similar structures at large scales, although the small scale features of the distributions do not overlap. This is in qualitative agreement with the argument discussed in Sec. II.

The scale-dependent colocalization is quantified by the cross probability $P_{12}(r)$ plotted in Fig. 3 for pairs of populations with different values of $\Delta \Psi$. We see that, for all pairs considered, the probability displays a scaling close to $r^{3}$ at very small scales confirming that, at these scales, the two populations have uncorrelated distributions. On the contrary,

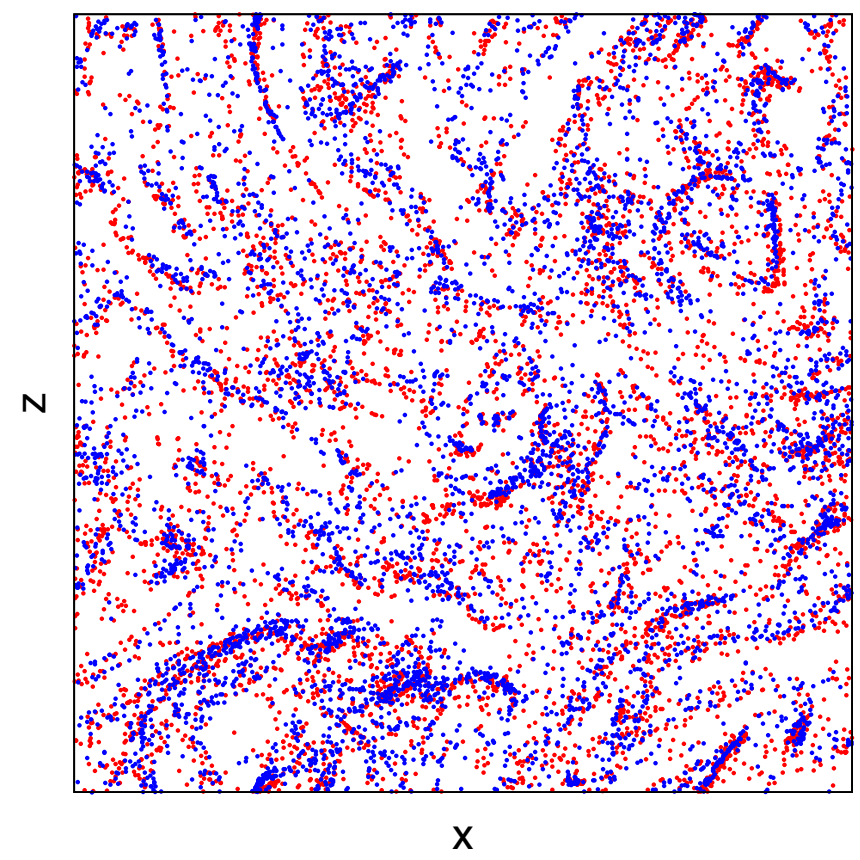

FIG. 2. Vertical section of the positions of two species of swimmers with $\Psi_{1}=0.5$ (red), $\Psi_{2}=0.667$ (blue), and $\Phi_{1}=\Phi_{2}=3.0$ in a turbulent flow.

for sufficiently large scales, the probability distribution follows a power-law scaling with exponent $\simeq 2.2$, close to the fractal dimension of a homogeneous population with stability number $\bar{\Psi}$, the average of the two species.

The transition between the two scaling ranges, although broad, clearly moves to larger scale as the difference $\Delta \Psi$ increases. In order to quantify this transition, we computed the crossover scale $R^{*}$ defined empirically by the intersection of two power-law fits of $P_{12}(r)$ at small scales and large scales, respectively. The small scale exponent is close to 3 (we obtain

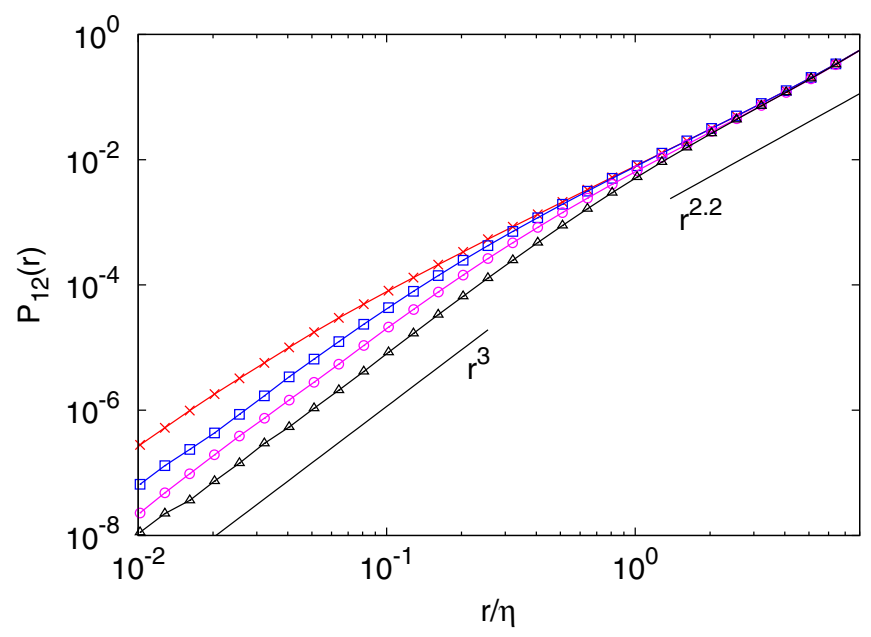

FIG. 3. Probability $P_{12}(r)$ to find two cells of different populations 1 and 2 at distance smaller than $r$ for different pairs of population parameters with $\Delta \Psi=0.0042$ (red crosses), $\Delta \Psi=0.021$ (blue squares), $\Delta \Psi=0.042$ (purple circles), and $\Delta \Psi=0.125$ (black triangles). Each population is composed by $6.4 \times 10^{4}$ individuals. 


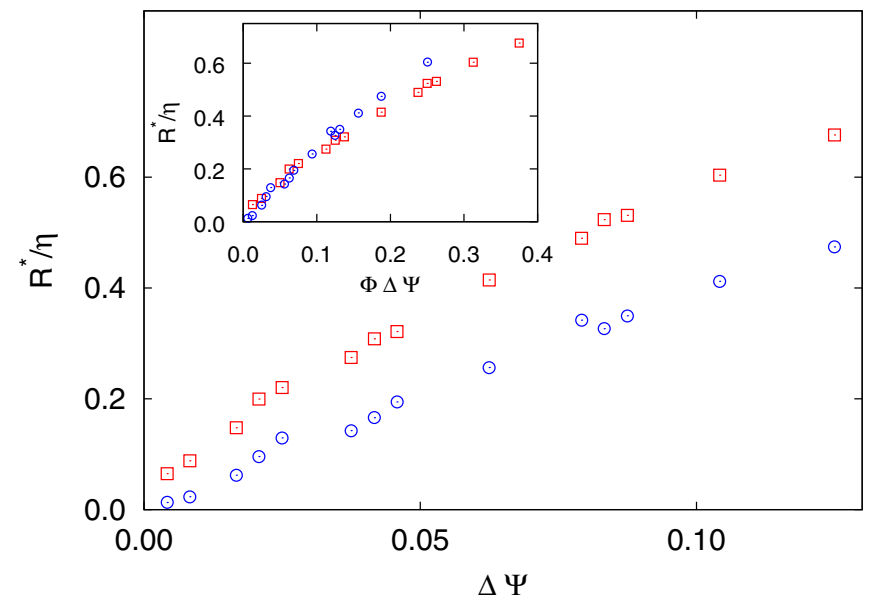

FIG. 4. Crossover scale $R^{*}$ as a function of $\Delta \Psi$ for two different sets of populations with $\Phi=3$ (red squares) and $\Phi=1.5$ (blue circles). (Inset) The same data plotted as a function of $\Phi \Delta \Psi$.

an exponent between 2.8 and 3.0 for all the cases considered), while the large-scale exponent depends on $\bar{\Psi}$. Figure 4 shows the dependence of $R^{*}$ on the population variance $\Delta \Psi$, for different swimming number $\Phi$, which confirms the linear scaling of $R^{*}$ predicted in Sec. II. The inset of Fig. 4 shows the remarkable collapse of $R^{*}$ when plotted as a function of $\Phi \Delta \Psi$, as predicted in Sec. II.

A similar behavior is observed when considering a bimodal population with two different swimming numbers $\Phi_{1}$ and $\Phi_{2}=\Phi_{1}+\Delta \Phi$. Figure 5 refers to three examples of bimodal populations characterized by three different stability numbers $\Psi$ close to the value for maximum clustering shown in Fig. $1(\Psi=0.4, \Psi=0.65$, and $\Psi=0.85)$. Also in this case, different scaling behaviors of $P_{12}(r)$ are observed for small and large separations and the fit of these scaling laws are used to define the crossover scale $R^{*}$ plotted in the figure. The inset of Fig. 5 shows a good collapse of the different crossover

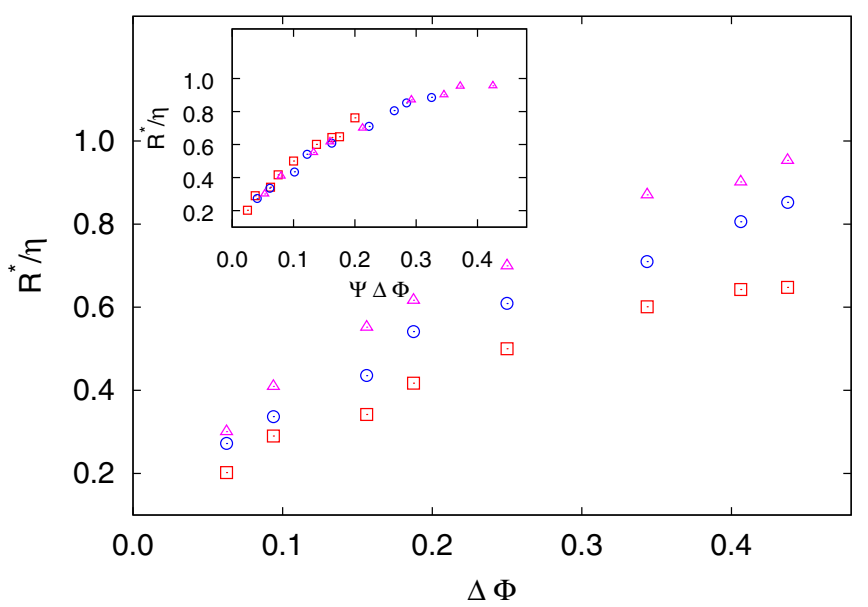

FIG. 5. Crossover scale $R^{*}$ as a function of $\Delta \Psi$ for two different sets of populations with $\Psi=0.4$ (red squares), $\Psi=0.65$ (blue circles), and $\Psi=0.85$ (pink triangles). (Inset) The same data plotted as a function of $\Psi \Delta \Phi$.

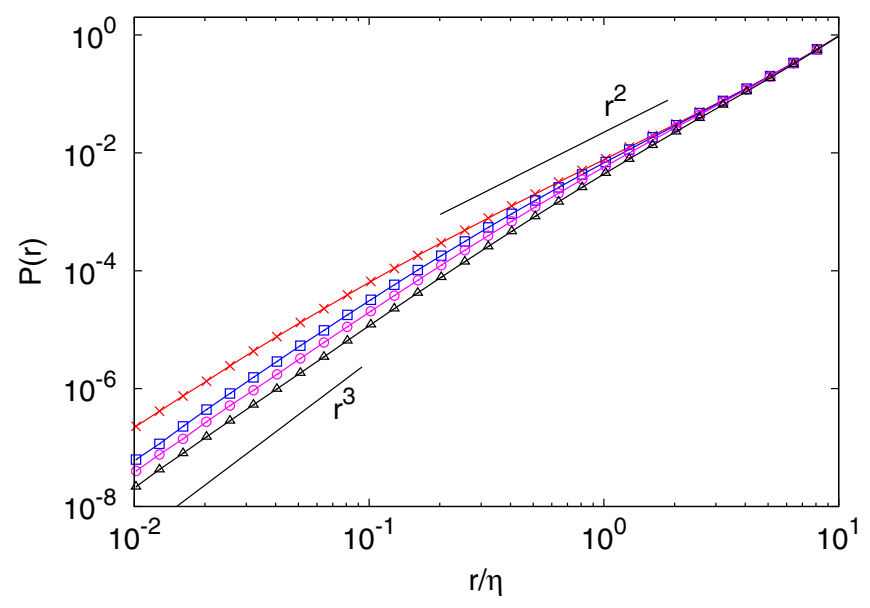

FIG. 6. Probability $P(r)$ to find two cells at distance smaller than $r$ for a population of gyrotactic swimmers with fixed $\Phi=3$ and $\Psi$ Gaussian distributed with $\bar{\Psi}=0.583$ and $\sigma_{\Psi}=0.008$ (red crosses), $\sigma_{\Psi}=0.042$ (blue squares), $\sigma_{\Psi}=0.083$ (purple circles), and $\sigma_{\Psi}=$ 0.166 (black triangles). Each population is composed by $3 \times 10^{5}$ individuals. (Inset) Crossover scale $R^{*}$ as a function of $\sigma_{\Psi}$.

scales when plotted as a function of the combination $\Phi \Delta \Psi$, confirming that this is the relevant parameter in the process.

\section{B. Gaussian distribution}

We now consider the more realistic case of a population of swimmers with stability number $\Psi$ following a Gaussian distribution with mean value $\bar{\Psi}$ and standard deviation $\sigma_{\Psi}$. Having in mind an experimental study in which we do not know the value of $\Psi$ (i.e., $B$ ) for each individual, we consider the cumulative probability $P(r)$ of having two cells at a distance smaller than $r$ integrated on all the possible pairs in the population.

The dependence of $P(r)$ on $r$ is shown in Fig. 6 for several populations with different standard deviations $\sigma_{\Psi}$. Similarly to the case of bimodal distribution, we recognize different ranges of scales. At very small scale, $P(r)$ converges towards the uniform scaling $r^{3}$, more clearly for the case with larger variance while for small variance a smaller scaling exponent is observed (between 2.6 and 2.8). At larger scales, $r \gtrsim \eta$, we observe a different power-law behavior with an exponent which weakly depends on $\sigma_{\Psi}$ and is very close to the exponent of a monodisperse population $D(\bar{\Psi}) \simeq 2$ for the smallest variance while grows to above 2.3 for the population with largest variance. As in the case of bimodal distribution, also in this case the transition from homogeneous $(D \simeq 3)$ to fractal ( $D \simeq 2$ ) distribution moves to larger scales as $\sigma_{\Psi}$ increases, as shown in Fig. 6. It is remarkable that also for the largest standard deviation, for which $\sigma_{\Psi} / \bar{\Psi} \simeq 0.29$, the distribution of the population at large scales is strongly inhomogeneous and the probability $P(r)$ indicates a fractal dimension close to $D(\bar{\Psi})$.

\section{CONCLUSIONS}

We have studied, by means of direct numerical simulations, the small-scale clustering of a population of gyrotactic cells, 
characterized by a distribution of gyrotactic parameters, swimming in a turbulent environment. The main goal of our work was to extend the results obtained for a monodisperse population to a more realistic population, characterized by a distribution of the swimming parameters.

We considered two very different families of test populations: bimodal populations, made of two hypothetical strains with different swimming or stability number, and a more realistic case where the swimming number is Gaussian distributed within the population. Despite the differences between the distributions considered, they show similar features for what concerns small-scale clustering. In all cases, the probability of finding interparticle distances less than $r$ exhibits two scaling ranges $r^{3}$ and $r^{D}$ for separations smaller and larger than a crossover scale $R^{*}$, respectively. The exponent $D$ represents the effective correlation dimension of the distribution when it is coarse grained at a scale $R^{*}$. The crossover scale grows with the variance of the distribution, confirming the linear dependence predicted for a narrow bimodal distribution. Furthermore, in this case our numerical data confirm the prediction that $R^{*}$ depends on the product of the two dimensionless swimming parameters.

From an experimental point of view, our results allow one to estimate a priori, based on biological and fluid-dynamical data, whether clustering is expected in a given range of scales for a given species. This should be taken into account in designing or analyzing field measurements in relation to turbulence-induced phytoplankton patchiness. Of course, analogous considerations apply every time fractal clustering is predicted, with a fractal dimension depending on parameters with a non-negligible intrinsic variability, as exemplified by works on inertial-particle transport in turbulence [26].

In this work we consider the limit of dilute concentration, i.e., we ignore possible effects due to direct interaction among cells and the feedback on the flow produced by cell motion.
These effects can be not negligible at high concentrations and in particular in the regions of fractal clustering where local density can be much larger than the average one [17]. On the basis of a model for the feedback force and torque induced by gyrotactic swimmers [28], one can estimate that the assumption of passive swimmers is valid up to concentrations of the order of $10^{6}$ cells $/ \mathrm{ml}$ which is hardly achieved in typical oceanic conditions.

Our findings should help assess the ecological relevance of turbulence-induced demixing [17]. Fractal clustering implies smaller distances between neighboring cells with respect to a homogeneous distribution with the same average density. This has consequences for mating, resource exploitation, and risk of predation. Consideration of the variability in swimming parameters might lead one to conclude that small-scale clustering is in practice irrelevant. However, if indeed the distribution is fractal on a finite range of scales, the effect on nearest-neighbor distance could be diminished but still relevant. If predation by zooplankton is considered, $R^{*}$ could be larger than the typical perception radius of the predator (e.g., a copepod or a fish larva), which would detect a locally homogeneous distribution of prey, but smaller than the typical swimming distances covered while cruising for prey, so that the underlying fractality might still have consequences for the predation strategy [29]. Moreover, the possibility of a heterogeneous population to retain a fractal distribution on larger scales may have effects for population dynamics [30,31].

\section{ACKNOWLEDGMENTS}

This article is based upon work from COST Action MP1305, supported by COST (European Cooperation in Science and Technology). We thank M. Cencini for fruitful discussion. R. Stocker is acknowledged for hospitality and useful discussions.
[1] R. G. Williams and M. J. Follows, Ocean Dynamics and the Carbon Cycle: Principles and Mechanisms (Cambridge University Press, Cambridge, 2011).

[2] J. Mitchell, H. Yamazaki, L. Seuront, F. Wolk, and H. Li, J. Mar. Syst. 69, 247 (2008).

[3] T. Kiørboe, A Mechanistic Approach to Plankton Ecology (Princeton University Press, Princeton, 2008).

[4] A. W. Visser and T. Kiørboe, Oecologia 148, 538 (2006).

[5] D. L. Mackas, K. L. Denman, and M. R. Abbott, Bull. Mar. Science 37, 652 (1985).

[6] A. Martin, Progr. Ocean. 57, 125 (2003).

[7] L. T. Mouritsen and K. Richardson, J. Plank. Res. 25, 783 (2003).

[8] E. Malkiel, O. Alquaddoomi, and J. Katz, Meas. Sci. Technol. 10, 1142 (1999).

[9] T. J. Pedley and J. O. Kessler, Proc. R. Soc. London B 231, 47 (1987); Annu. Rev. Fluid Mech. 24, 313 (1992).

[10] W. M. Durham, J. O. Kessler, and R. Stocker, Science 323, 1067 (2009).

[11] J. O. Kessler, Nature (London) 313, 218 (1985).

[12] G. J. Thorn and R. N. Bearon, Phys. Fluids 22, 041902 (2010).
[13] S. O’Malley and M. A. Bees, Bull. Math. Biol. 74, 232 (2012).

[14] D. M. Lewis, Proc. R. Soc. London A 459, 1293 (2003).

[15] W. M. Durham and R. Stocker, Annu. Rev. Mar. Sci. 4, 177 (2012).

[16] F. Santamaria, F. De Lillo, M. Cencini, and G. Boffetta, Phys. Fluids 26, 111901 (2014).

[17] W. M. Durham, E. Climent, M. Barry, F. De Lillo, G. Boffetta, M. Cencini, and R. Stocker, Nature Comm. 4, 2148 (2013).

[18] F. De Lillo, M. Cencini, W. M. Durham, M. Barry, R. Stocker, E. Climent, and G. Boffetta, Phys. Rev. Lett. 112, 044502 (2014).

[19] C. Zhan, G. Sardina, E. Lushi, and L. Brandt, J. Fluid Mech. 739, 22 (2013).

[20] K. Gustavsson, F. Berglund, P. R. Jonsson, and B. Mehlig, Phys. Rev. Lett. 116, 108104 (2016).

[21] K. Gustavsson and B. Mehlig, Advan. Phys. 65, 1 (2016).

[22] T. Pedley and J. Kessler, Annu. Rev. Fluid Mech. 24, 313 (1992).

[23] U. Frisch, Turbulence: The Legacy of AN Kolmogorov (Cambridge University Press, Cambridge, 1995).

[24] G. Paladin and A. Vulpiani, Phys. Rep. 156, 147 (1987).

[25] J. Bec, J. Fluid Mech. 528, 255 (2005). 
[26] J. Bec, A. Celani, M. Cencini, and S. Musacchio, Phys. Fluids 17, 073301 (2005).

[27] L. Biferale, G. Boffetta, A. Celani, B. J. Devenish, A. Lanotte, and F. Toschi, Phys. Rev. Lett. 93, 064502 (2004).

[28] T. Pedley and J. Kessler, J. Fluid Mech. 212, 155 (1990).

[29] D. W. Sims, E. J. Southall, N. E. Humphries, G. C. Hays, C. J. Bradshaw, J. W. Pitchford, A. James, M. Z. Ahmed,
A. S. Brierley, and M. A. Hindell, Nature (London) 451, 1098 (2008).

[30] R. Benzi, M. H. Jensen, D. R. Nelson, P. Perlekar, S. Pigolotti, and F. Toschi, Eur. Phys. J. Special Topics 204, 57 (2012).

[31] S. Pigolotti, R. Benzi, M. H. Jensen, and D. R. Nelson, Phys. Rev. Lett. 108, 128102 (2012). 